human achievement, and that what distinguishes language from the communication systems of our primate cousins is grammar. Grammars of natural languages signal grammatical relations, using word order or cases. They have tense endings, agreement markers, gender, distinctions between singular and plural, personal pronoun systems and so on. Most human infants learn these complex systems perfectly, without apparent effort. Research has concentrated more on the form of the infant's acquisition of the abstract linguistic code than on comparisons with nonhuman primates, precursors to language in the child's very early development, or the biological or social context of language development.

Locke's aim is to redress this balance by close scrutiny of the first year of life. In a fascinating, scholarly and clearly written account, he takes us to the brink of language proper by examining its perceptual, social, neural and cognitive precursors from before birth to the appearance of the first recognizable words. What is it about the human infant that makes it different from the young nonhuman primate? And what is it about the strands of early development, and the social context in which they are embedded, that guarantees that all but a small percentage of human infants are eventually able to speak the language of their environment?

Much of the material brought together in the book - the development of the child's early ability to recognize faces and voices, the development of perceptual capabilities, vocal behaviour and neural capacity - is not new. What is original is the unifying perspective from which Locke synthesizes a mass of information from his own and others' previous research. The question he wishes to address is how a child comes to speak. This ontogenetic equivalent of a phylogenetic question that we can never answer demands a systematic review of the development in the child's first year of a "variety of language-related mechanisms [which] will inch their way toward efficient action". As a source of information on these mechanisms, this book would be difficult to better. But a detailed description of the increasing complexity of the child's vocal behaviour, its developing perceptual abilities and capacity for reference does not fully account for the fact that children learning any language begin to use words from their parents' lexicons at or about the time of their first birthdays.

The author's explanation for the infant's motivation to speak fully acknowledges the role of language as a medium for social and emotional interaction. Language expresses affect (emotion associated with an idea) through prosody (patterns of stress and intonation), while also expressing propositions through words and their grammatical organization. Locke believes that these dimensions of language, the "warmly emotional" and the "coldly analytical", are equally important. Indeed, for the infant, the affective dimension may well be initially more important. Locke argues that it is the infant's social cognition, in relation to what is said to him or her, that plays a "precursive and enabling role" in the development of spoken language. This (possibly specialized) capability is seen, for example, in the neonate's orientation to face-like stimuli a few minutes after birth, the gazing of infant and mother at one another, and early infant preferences for the mother's voice and prosody. The infant therefore does not 'acquire' language but is drawn towards it by his or her active desire to communicate with caretakers, and by their reciprocal role in socialization. The specialization in social cognition, some aspects of which are shared with nonhuman primates, delivers the infant to the point at which the other aspect of our species' dual specialization for language, the grammatical analysis module, can contribute.

While impressively developing his thesis, Locke is careful to bring the child only as far as the 'doorstep' of theories about the acquisition of the abstract linguistic code. There is little doubt that his bio-linguistic perspective on the precursors of linguistic development will influence researchers working on that subject. I would also hope that his work will encourage researchers examining early grammatical and phonological development to consider seriously the biological basis of these aspects too.

Paul Fletcher is in the Department of Linguistic Science, University of Reading, PO Box 218, Reading RG6 2AA, UK.

\title{
Warm or cold environments?
}

\section{Eric J. Barron}

Climate Modes of the Phanerozoic. By L. A. Frakes, J. E. Francis and J. I. Syktus. Cambridge University Press: 1992. Pp. 274. £40, \$69.95.

CLIMATE Modes of the Phanerozoic is a review of the Earth's climate over the past 600 million years. This text is an update of an earlier work by L. A. Frakes, Climates Throughout Geologic Time (Elsevier, 1979). There are some important differences. Whereas the earlier text examined climate as a function of stratigraphic subdivisions of time, the organizing basis of the new text is the warm and cold climate 'modes' of the Phanerozoic. The authors emphasize the climatic extremes recorded in rocks and sediments and describe the entire record as sets of either cold or warm climates. The new volume is also strengthened by the addition of material on the terrestrial climate record and a greater emphasis on Quaternary climates.

There is little discussion about the continuing controversy over climate forcing factors, modes or the characteristics of past climates. The book is primarily an interpretation of the climatic record based on data reconstructions and correlations with key indicators. As such, the text is likely to spark debate and controversy at several levels. For example, many researchers today emphasize the differences of the warm climates during the Phanerozoic as opposed to the similarities. These workers are likely to view the modes as artificial distinctions or missing important climate information.

The Pliocene warm interval, for in- stance, is the focus of international efforts to understand warm climates, yet it is characterized as part of a cold mode by the authors. The literature on the Early Eocene emphasizes a debate on whether this warm interval may be explained by a change in the role of ocean heat transport. Such a change would produce a climate very different from that of the Late Cretaceous. Yet the Late Cretaceous and the Early Eocene are described as part of one warm climate mode in the text.

The interpretation of particular climatic characteristics will also provoke debate. The authors describe the Late Cretaceous circulation as sluggish. Is this reasonable? Can we maintain warm poles with a weak atmospheric and oceanic circulation? The authors link the occurrence of evaporites to temperature. Is temperature alone a good predictor of the moisture balance? Does the restriction of seaways in the subtropics play a critical role? The authors introduce the possibility that the galactic year - the time taken by the Sun to orbit the centre of the Milky Way - may be correlated with the climate modes. Can this be tested and can we define a plausible mechanism that justifies this correlation?

As an interpretation of the climatic record, this book will prompt a lot of discussion because much of the interpretation is associated with uncertainty. But it is the most definitive reference so far on the interpretation of the climatic evolution of Earth as a series of alternating warm and cold modes.

Eric J. Barron is at the Earth System Science Center, Pennsylvania State University, University Park, Pennsylvania 16802, USA. 